\title{
Scanning Electron Microscopy and Atomic Force Microscopy of the Ring Structures in Human Calcium Oxalate Urinary Stones
}

\author{
Peter Rez* , Hanson Fong** and Mehmet Sarikaya** \\ *Department of Physics and Astronomy and CSSS, Arizona State University, Tempe, AZ 8528 \\ **Department of Materials Science and Engineering, University of Washington, Seattle, WA 98195
}

Kidney stones are a painful affliction that will affect almost $10 \%$ of the population at some time during their lifetimes. More than $50 \%$ of the stones formed by adults in industrialised countries have calcium oxalate as their major constituent. Both the monohydrate form of calcium oxalate known as whewellite, and the dihydrate form, mineral name wedellite, have been found in human kidney stones. Calcium oxalate dihydrate forms distinctive bipyramids and is usually found on the outside of mixed stones. The mechanism for kidney stone formation is still controversial and the role of various promoters or inhibitors in the growth and aggregation of crystallites is still the subject of ongoing research.

When a calcium oxalate monohydrate stone is sectioned optical microscopy shows distinct rings with radial striations surrounding a nucleus[1]. (See Fig 1). Our earlier work[2], using both AFM and Low voltage SEM, showed that stones are loosely ordered arrangements of crystallites ranging in size from $500 \AA$ to $3000 \AA$. To determine the microstructural origins of the ring structures six stones were selected after analysis by XRD confirmed their overall composition. The stones were sectioned following the procedure described by Shaapur et al [3], but the final of microtomy was omitted. Both the SEM and AFM images showed the crystallites found in earlier work. Fig 2 shows the same area imaged using AFM and SEM showing the similarity in contrast. Both AFM and SEM measure height above a reference surface though the AFM appears to have better resolution and, as expected, the SEM has greater depth of field.

Rings structures from the box shown in Fig 1 were imaged using bath AFM (Fig 3) and SEM (Fig 4). The darker portions of the ring appear to be lower and to have a lower density of smaller particles, while the lighter regions appear to be higher and have a high density of larger particles. In ring structures from another area of the same stone the crystallites were elongated and differed in length. In other stones changes in the alignment of crystallites were observed. These observations are consistent with the optical microscopy. Light scattering from the smaller crystallites would be lower giving a darker contrast. Also any changes in orientation would be especially noticeable when viewing with polarized light. Although the rings are an intrinsic property of the stone, the preparation techniques make them detectable since the smaller crystallites in lower density regions are more easily removed. The physiological cause of different size distributions is still unknown, though if stones are similar to other biominerals there might be some sub-ellular structure in which mineral formation takes place.

\section{References}

[1] E.L. Prien and E.L. Prien, Amer. J. Medicine 45 (1968) 654.

[2] H.H. Dorian, P. Rez and G.W. Drach, J. Urol. 156 (1996) 1833.

[3] F. Shaapur, P Rez, D.R. Yaniv and G.W. Drach, Microsc. Soc. America (1994) 884. 


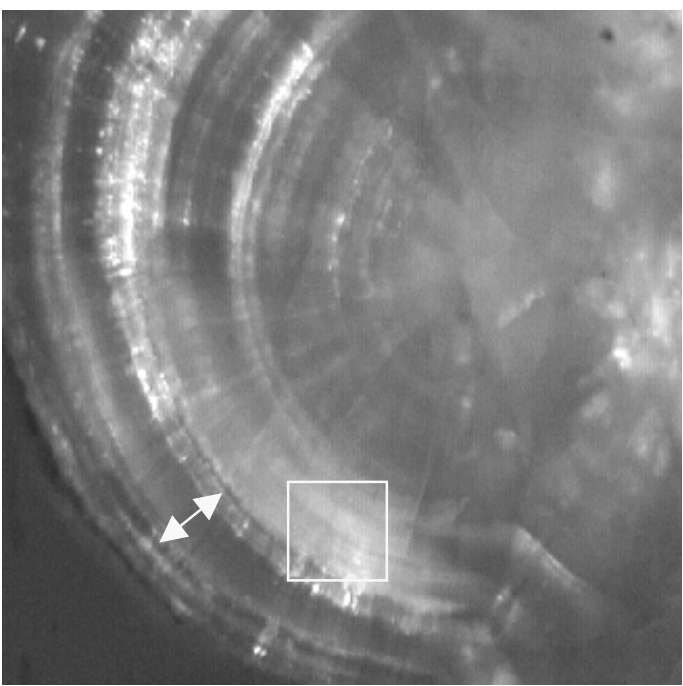

FIG 1.Optical micrograph of monohydrate stone Bar is $0.1 \mathrm{~mm}$
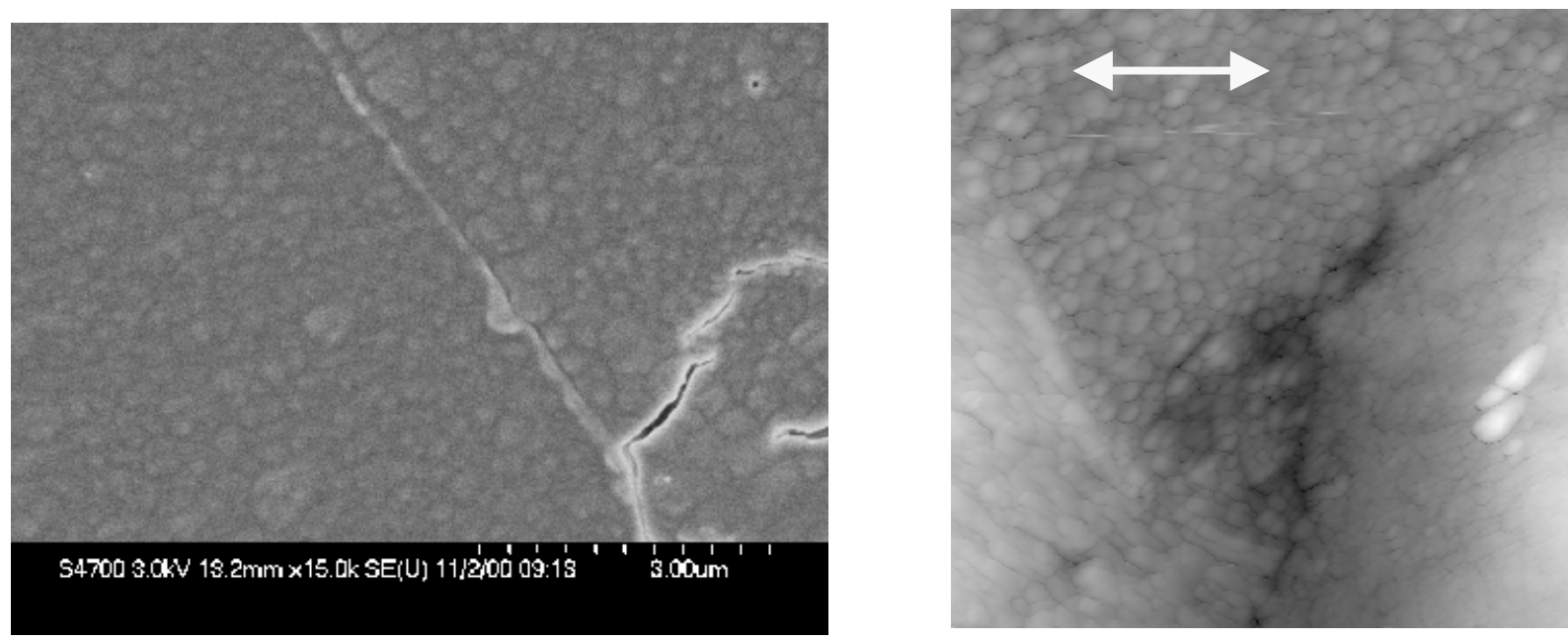

FIG 2(a) SEM view of different monohydrate stone

FIG 2(b) AFM view Bar is $1 \mu \mathrm{m}$
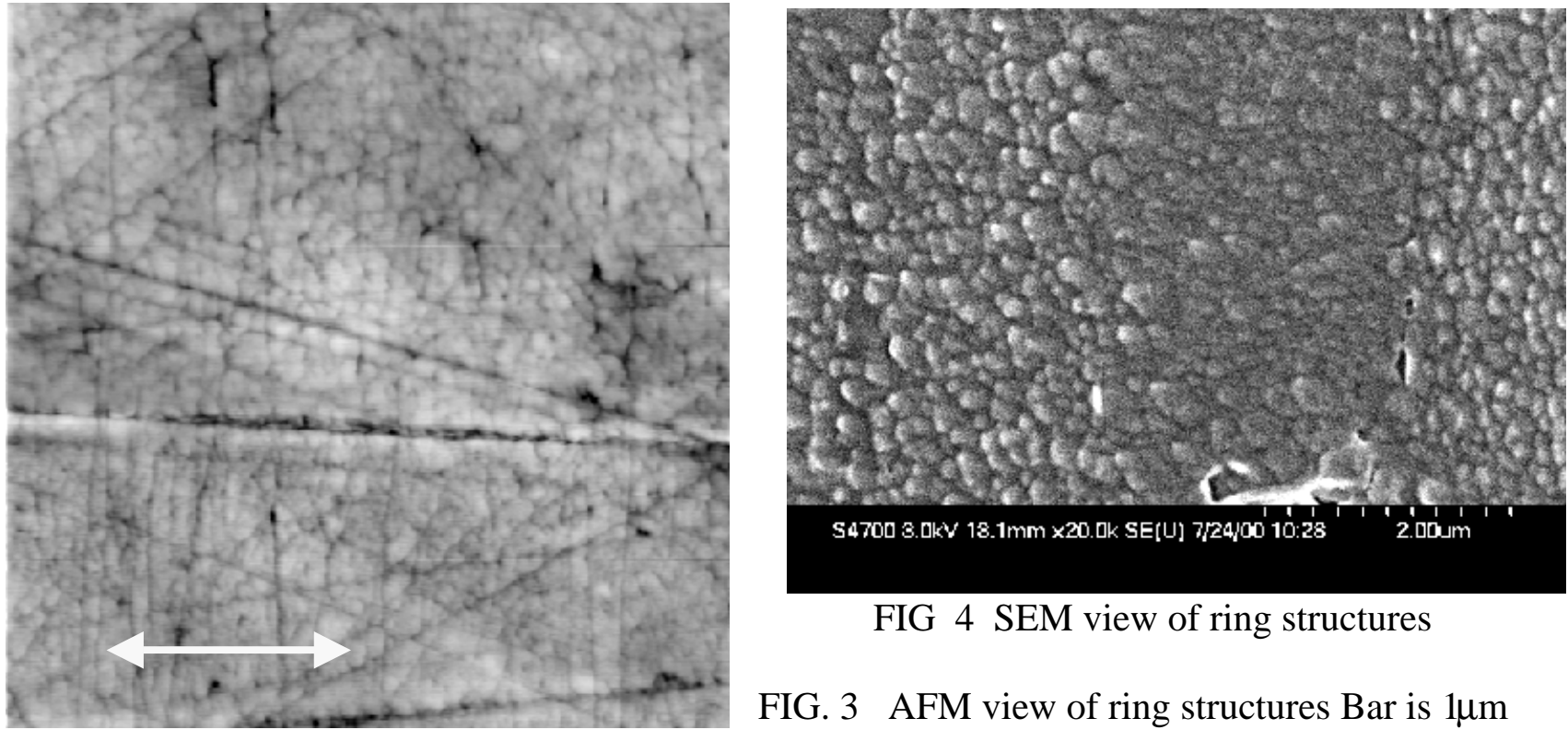

FIG 4 SEM view of ring structures

FIG. 3 AFM view of ring structures Bar is $1 \mu \mathrm{m}$ 